\title{
THE OPEN CASE FOR SIMPLE ALTERNATIVE RINGS
}

\author{
M. SLATER
}

1. Introduction. Let $R$ be a simple alternative ring, not associative, and not a Cayley-Dickson algebra over its center. It was proved by Kleinfeld ([2] and [3]) that $R$ is necessarily nil and of char 3. It has remained an open question whether such a ring $R$ can exist.

We show here, using a result given by Shirshov (in [6]; see also [5]), that such a ring cannot exist (Theorem C).

2. Shirshov's result. We now state a convenient version of one of Shirshov's theorems. Let $X$ be any set (of 'indeterminates'). Let $N_{X}(F)$ and $S_{X}(F)$ be, respectively, the free nonassociative and free associative algebras over the field $F$ and on the free generating set $X$. There is an obvious $F$-homomorphism $\alpha$ from $N_{X}(F)$ onto $S_{X}(F)$ : in the standard representation $\alpha$ deletes all parentheses. Say $p \in N_{X}(F)$ is admissible provided $p \alpha \neq 0$.

Now suppose $R$ is any (nonassociative) algebra over $F$. Say $R$ satisfies the admissible polynomial identity (p.i.) $p$, provided $p \in N_{X}(F)$ is admissible, and for every $F$-homomorphism $\beta$ of $N_{X}(F)$ into $R$, $p \beta=0$.

We can now state

ThEOREM A (ShIRshov). ${ }^{1}$ Let $R$ be an alternative algebra over a field $F$ of char $\neq 2$. Suppose $R$ satisfies an admissible p.i. If $R$ is algebraic over $F$, then $R$ is locally finite over $F$.

CoRollary 1. Under the hypotheses of Theorem $A$, if $R$ is nil, then $R$ is locally nilpotent.

Proof. Let $T$ be any finitely generated subalgebra of $R$. Then by Theorem A, $T$ is finite dimensional over $F$. But it is a classical result (e.g. see $[4$, p. 30]) that a nil finite-dimensional alternative algebra is nilpotent. So $T$ is nilpotent, and $R$ is locally nilpotent.

In view of Corollary 1 , we now turn our attention to locally nilpotent rings.

Received by the editors March 20, 1967.

1 The relevant result is Theorem 5 of [6]. The statement of this theorem contains some errors, and the proof outlined is rather obscure. A set of notes giving a full exposition of the relevant results has been prepared by the author of the present paper, and is available from him. 
3. Locally nilpotent rings. Let $R$ be either a ring or an algebra over a field $F . R$ need not be associative. We say $R$ is locally nilpotent provided every finitely generated subring (respectively subalgebra) is nilpotent.

Lemma 2. Let $R$ be a locally nilpotent ring or algebra. Given $a \in R$, let $A$ be the ideal of $R$ generated by $a R+R a$. If $a \in A$, then $a=0$.

Proof. We first introduce some notation. Let $E(R)$ be the endomorphism ring (or algebra) of $R$. Given $s \in R$, we define $L(s)$ and $R(s) \in E(R)$ by $x \cdot R(s)=x s ; x \cdot L(s)=s x$ (all $x \in R$ ). We write $U(s)$ to denote ambiguously either $R(s)$ or $L(s)$.

If $(s, n)=\left(s_{1}, s_{2}, \cdots, s_{n}\right)$ is an $n$-tuple of elements of $R(n \geqq 1)$, we write $U[s, n]$ for any $V \in E(R)$ of the form $V=U\left(s_{1}\right) \cdot U\left(s_{2}\right)$ $\cdots \cdot U\left(s_{n}\right)$. Thus $V$ is the product of $n$ right or left multiplications.

We define addition on the set of tuples by setting $(s, n)+\left(s^{\prime}, m\right)$ $=\left(s_{1}, s_{2}, \cdots, s_{n}, s_{1}^{\prime}, s_{2}^{\prime}, \cdots, s_{m}^{\prime}\right)=\left(s+s^{\prime}, n+m\right)$, say. Then it is immediate that any product $U[s, n] \cdot U\left[s^{\prime}, m\right]$ is some $U\left[s+s^{\prime}, n+m\right]$.

Now in any ring or algebra $R$ the ideal generated by $a R+R a$ (for given $a \in R$ ) is easily seen to be the set of all finite sums of the form

$$
\sum_{i} a \cdot U[s(i), n(i)], \quad n(i) \geqq 1 ;(s(i), n(i))=\left(s(i)_{1}, \cdots, s(i)_{n(i)}\right) .
$$

In particular, if $a \in A$, we can write

$$
a=\sum_{i=1}^{r} a \cdot U[s(i), n(i)], \quad n(i) \geqq 1 .
$$

Set

$$
T=\{a\} \cup\left\{s(i)_{j(i)}: 1 \leqq j(i) \leqq n(i) ; 1 \leqq i \leqq r\right\} .
$$

We now claim that for any given $m \geqq 1$ we can write $a$ in the form

$$
a=\sum_{i=1}^{t} a \cdot U[s(i), n(i)], \quad n(i) \geqq m,
$$

where $t=t_{m}, s(i)=s_{m}(i), n(i)=n_{m}(i)$ are all functions of $m$, and every $s(i)_{j(i)} \in T$.

For $m=1$ such an expression is given in (i) (by definition of $T$ ). We prove the general assertion by induction on $m$. Suppose we have an expression (ii) for $a$ and given $m \geqq 1$. Then, substituting the expression (ii) for $a$ in the right side of the equality (ii), we obtain 


$$
\begin{aligned}
a & =\sum_{i=1}^{t}\left\{\sum_{j=1}^{t} a \cdot U[s(j), n(j)]\right\} \cdot U[s(i), n(i)] \\
& =\sum_{i=1}^{t} \sum_{j=1}^{t} a \cdot\{U[s(j), n(j)] \cdot U[s(i), n(i)]\} \\
& =\sum_{i, j=1}^{t} a \cdot U[s(j)+s(i), n(j)+n(i)] .
\end{aligned}
$$

Since $n(j)+n(i) \geqq m+m \geqq m+1$, this expression for $a$ is of the form (ii) with $m+1$ in place of $m$. This completes the induction.

Now let $S$ be the subring or subalgebra of $R$ generated by $T$. Since $T$ is a finite set, $S$ is nilpotent, say of index $m$. If $a \in A$, then by (ii) we can write $a$ as a sum of terms $a \cdot U[s, n] ; n \geqq m$. But $a \cdot U[s, n]$ is a product of $\geqq n+1 \geqq m$ elements of $T$, and so lies in $S^{m}=(0)$. Thus $a$ is a sum of zero terms, and $a=0$.

As an easy corollary we have

TheOREм B. Let $R$ be a simple (nonassociative) ring. Then $R$ is not locally nilpotent.

Proof. Suppose $R$ is simple and locally nilpotent. Let $0 \neq a \in R$ be arbitrary, and $A$ as in Lemma 2. Then $a \notin A$, so $A \neq R$, whence $A=(0)$. Thus $a$ is a total zero-divisor. So $R$ consists entirely of zerodivisors, and thus is a zero ring. Such rings are not regarded as being simple.

Note. This proof was inspired by that given in $[1$, p. 31$]$ for associative rings.

4. Simple alternative rings. We start by recalling some known results. They may all be found in [3]. Let $R$ be an alternative ring, $N(R)$ its nucleus, $Z(R)$ its center. For $x, y \in R$, write $(x, y)$ for $x y-y x$. Then we have

Lemma 3 (Kleinfeld). For all $x, y \in R,(x, y)^{4} \in N(R)$. If $R$ is prime, $N(R)=Z(R)$. If $R$ is simple, but neither associative nor a Cayley-Dickson algebra over $Z(R)$, then $3 R=(0)$ and $R$ is nil.

We now have

THEOREM C. If $R$ is a simple alternative ring, then $R$ is either associative or a Cayley-Dickson algebra over $Z(R)$.

Proof. Suppose $R$ is neither. Then by Lemma 3 we may regard $R$ as a nil algebra over the field $J_{3}$ of char $\neq 2$. Since simple rings are 
prime, it also follows from Lemma 3 that $R$ satisfies the identity $p=p(x, y, z)=\left((x, y)^{2}(x, y)^{2}, z\right) \in N_{X}\left(J_{3}\right)$. Since $p$ is admissible, $R$ is locally nilpotent by Corollary 1 . By Theorem $\mathrm{B}$ such an $R$ cannot exist.

Acknowledgment. I would like to thank Professors Kleinfeld and Shirshov for a conversation (during the Moscow Congress) at which the existence of [5] became known to me.

\section{REFERENCES}

1. I. N. Herstein, Ring theory, Mimeographed notes, Univ. of Chicago, Chicago, 1961.

2. E. Kleinfeld, Simple alternative rings, Ann. of Math. (2) 58 (1953), 544-547.

3. - Alternative nil rings, Ann. of Math. (2) 66 (1957), 395-399.

4. R. D. Schafer, An introduction to nonassociative algebras, Academic Press, New York, 1966.

5. A. I. Shirshov, On some non-associative nil rings and algebraic algebras, Mat. Sb. (N.S.) (83) 41 (1957), 381-395. (Russian)

6. - On rings with identical relations, Mat. Sb. (N.S.) (85) 43 (1957), 277283. (Russian)

University of Bristol, England and

UNIVERSITY OF HAWAII 